\title{
RBP and Alb in neonatal hydronephrosis and its association with IL-12 levels in pregnant women before delivery
}

\author{
ZHI MA ${ }^{1}$, DEZHI LU ${ }^{1}$, WANXIN ZHAO ${ }^{2}$ and HUIZHI WANG ${ }^{3}$ \\ ${ }^{1}$ Department of Pediatric Surgery, Hongqi Hospital Affiliated to Mudanjiang Medical University; \\ ${ }^{2}$ Department of Gynaecology and Obstetrics, Second Affiliated Hospital of Mudanjiang Medical University; \\ ${ }^{3}$ Department of Gynaecology and Obstetrics, Hongqi Hospital \\ Affiliated to Mudanjiang Medical University, Mudanjiang, Heilongjiang 157000, P.R. China
}

Received November 28, 2018; Accepted April 19, 2019

DOI: $10.3892 / e t m .2019 .7605$

\begin{abstract}
Expression levels of retinol-binding protein (RBP) and albumin (Alb) in the kidney of patients with neonatal hydronephrosis were detected to explore whether RBP and Alb can be used as diagnostic indicators for neonatal hydronephrosis. Blood and urine samples from neonates with hydronephrosis in the obstetrics and gynaecology department of Hongqi Hospital Affiliated to Mudanjiang Medical University from January 2016 to January 2018, and healthy newborns were collected. RBP and Alb levels in neonates, and the expression of IL-12 in serum of the mothers were detected by ELISA. To establish the rat model, 30 male Sprague Dawley (SD) rats were randomly divided into two groups: sham operation group $(\mathrm{n}=15)$ and unilateral ureteral obstruction (UUO) $(n=15)$. Expression of RBP and Alb protein was detected by western blot analysis. Another 30 female SD rats were randomly divided into the Sham and IL-12 knockdown groups. The Sham group was given NC treatment, while IL-12 knockdown group was given treatment with IL-12 knockdown adenovirus through tail vein injection. Results of ELISA showed that serum RBP and renal urinary Alb levels were higher in neonates with hydronephrosis compared with the control group $(\mathrm{P}=0.009$ or $\mathrm{P}=0.013)$. Compared with the control group, levels of IL-12 were significantly lower in mother of neonatal hydronephrosis group $(\mathrm{P}=0.001)$. After the IL-12 knockdown treatment in pregnant rats, levels of RBP and Alb hydronephrosis-related indicators were significantly increased. The incidence of hydronephrosis in rats delivered via IL-12 knockdown pregnant rats was significantly higher than that of normal pregnant rats $(\mathrm{P}=0.001)$. The combination
\end{abstract}

Correspondence to: Dr Huizhi Wang, Department of Gynaecology and Obstetrics, Hongqi Hospital Affiliated to Mudanjiang Medical University, 5 Tongxiang Road, Aimin, Mudanjiang, Heilongjiang 157000, P.R. China

E-mail: mazhi913@126.com

Key words: neonatal hydronephrosis, retinol binding protein, albumin, interleukin-12 of RBP and Alb in the diagnosis of neonatal hydronephrosis has important clinical significance, and neonatal hydronephrosis is closely related to the level of IL-12 in mothers before birth. Our findings provide new ideas and theoretical basis for the diagnosis and prediction of neonatal hydronephrosis.

\section{Introduction}

In recent years, with the widespread concern about neonatal medicine, neonatal medicine has developed rapidly, and studies on neonatal diseases have attracted increasing attention. Hydronephrosis is one of the common diseases in urology. Neonatal hydronephrosis is considered as the most controversial and relatively difficult type of hydronephrosis (1). According to statistical analyses, neonatal hydronephrosis is the most common genitourinary disease in children. The stenosis of the junction of the fistula, posterior urethral valve, multiple cysts of the kidney, ureteral cyst, lower ureteral obstruction, vesicoureteral reflux and other causes lead to hydronephrosis in newborns (2-4). International neonatal urology association proposed that separation of the renal collecting system more than $5 \mathrm{~mm}$ before 24 weeks or more than $10 \mathrm{~mm}$ after 24 weeks was used as the standard for the diagnosis of neonatal hydronephrosis (5). At present, study on the diagnosis and pathological evolution of neonatal hydronephrosis is not complete and has great controversy (6). Perinatal ultrasound is used to diagnose fetal hydronephrosis, but it lack accuracy to some extent because of the easy interference of gas in the gastrointestinal tract.

Retinoid-binding protein (RBP) is a lipophilic carrier protein synthesized by the rough endoplasmic reticulum of hepatocytes, with a small molecular weight and widely distributed in human body fluids. Albumin (Alb) is a protein that can be filtered through the glomerular filtration membrane and can be reabsorbed by the renal tubules. Hao et al detected the levels of Alb and RBP in kidney of the affected kidney of rabbit model of hydronephrosis. It was found that the levels of RBP and Alb in urine were significantly increased when renal function was impaired. It was concluded that RBP and Alb can be used as indicators for diagnosing the degree of renal dysfunction of hydronephrosis (7-9). At present, there are no studies reported that the combined detection of these 
two indicators can be used for the diagnosis of neonatal hydronephrosis. In addition, interleukin-12 (IL-12) is a Th1 type cytokine produced by macrophages and B cells and capable of participating in immune regulation and promoting Th1 type cell differentiation. Liu et al found that IL-12 played a very important regulatory role in inflammation and infection response (10). Therefore, we speculate that neonatal hydronephrosis may be associated with inflammatory infections and the decreased IL-12 in pregnant women.

In this study, we investigated the levels of RBP and Alb in kidney of patients with neonatal hydronephrosis, and explored the diagnosis and values of combined detection of RBP and Alb for neonatal hydronephrosis. Through a rat model, the accuracy and sensitivity of combined detection of RBP and Alb for neonatal hydronephrosis were preliminarily investigated, which provided a more accurate method for clinical diagnosis of neonatal hydronephrosis. This study further clarified neonatal hydronephrosis and provided theoretical knowledge and experimental basis for clinical treatment of hydronephrosis.

\section{Materials and methods}

General Information. Pregnant women $(n=24$, mean age: $30.15 \pm 6.32$ years) who gave a birth in Hongqi Hospital Affiliated to Mudanjiang Medical University (Mudanjiang, China) from January 2016 to January 2018 were selected as the study subjects. Pregnant women were monitored until the third trimester and only the ones with complete medical record were included. Inclusion criteria were: Prenatal diagnosis of hydronephrosis and no drainage during pregnancy; meeting the diagnostic criteria of fetal hydronephrosis; pregnant women who had no physical discomfort and no other genetic diseases; patients who signed an informed consent. This study excluded patients with a history of teratogenesis, adverse pregnancy and childbirth history, and incomplete clinical data.

We determined the presence of hydronephrosis in the fetus through the perinatal data of the pregnant woman before delivery. One week before the birth of the fetus, pregnant women fasted for 12-14 $\mathrm{h}$ and $5 \mathrm{ml}$ of venous blood was collected in the morning. After the birth of newborns, blood and urine samples were collected. A total of 48 neonatal samples were collected. Of these, 24 neonates (13 males and 11 females) with hydronephrosis were the disease group. In the disease group, the mean gestational age was $36.79 \pm 3.01$ weeks, the mean age was $16.25 \pm 7.25$ days, the mean birth weight was $3584.04 \pm 617.76$ grams, and the mean body mass index was $22.11 \pm 2.21 \mathrm{~kg} / \mathrm{m}^{2}$. All children in the neonatal hydronephrosis group were unilateral hydronephrosis (children with bilateral hydronephrosis were not included in the study), and ultrasound showed anteroposterior diameter $(A P D) \geq 7 \mathrm{~mm}$. According to the grading criteria proposed by Grignon et al (11) the neonatal hydronephrosis was classified into 5 grades: grade I (APD $<10 \mathrm{~mm}$ ), grade II (APD $>10 \mathrm{~mm}-<15 \mathrm{~mm}$ ), grade III (APD $>15 \mathrm{~mm}$ ) with mild renal pelvis expansion, grade IV (APD $>15 \mathrm{~mm}$ ) with moderate renal dilatation and grade $\mathrm{V}(\mathrm{APD}>15 \mathrm{~mm})$ with severe renal dilatation $(11,12)$. In this study, there were 5 cases of grade I, 5 of grade II, 5 of grade III, 5 of IV, and 4 of grade V. During the same time period, another 24 healthy newborns (10 males and 14 females) were enrolled to serve as the control group. In control group, the mean gestational age was $36.77 \pm 3.40$ weeks, the mean age was $17.96 \pm 7.81$ days, the mean birth weight was $3607.5 \pm 630.16$ grams, and the mean body mass index was $22.01 \pm 2.83 \mathrm{~kg} / \mathrm{m}^{2}$. There was no significant difference in sex, age, gestational age, birth weight, or body mass mean index between the two groups, with no statistical significance ( $P>0.05$ ) (data not shown). Thus, follow-up studies could be carried out.

Exclusion criteria: urinary tract infection, fever, ketoacidosis, heart failure, hypertension, diabetes, urinary tract or other kidney disease, kidney malformation, serum creatinine $\geq 177.0 \mathrm{mmol} / \mathrm{l}$ and the use of kidney damage drugs.

All the experimental procedures were approved by the patients' families and pregnant women. All participants or their parents signed an informed consent. This study was approved by the Medical Ethics Committee of Hongqi Hospital Affiliated to Mudanjiang Medical University.

Detection of IL-12 in blood samples of pregnant women. The blood IL-12 level of pregnant women was detected by using an immunoenzyme-linked immunosorbent assay (ELISA) kit (Cloud-Clone Corp., Houston, TX, USA). Operations were carried out in strict accordance with the instructions. Absorbance values were measured at $450 \mathrm{~nm}$ using a microplate reader (Tecan Group, Ltd.).

Collection of newborn samples and detection of RBP and Alb. Neonatal venous blood $(5 \mathrm{ml})$ was centrifuged at 2,000 x $\mathrm{g}$ for $10 \mathrm{~min}$ at room temperature, and the supernatant was taken. RBP was detected using a kit provided by Shanghai Beijia Biochemical Reagent Co., Ltd. and 7180 Hitachi automatic biochemical analyzer, and the remaining serum was placed in a refrigerator at $-80^{\circ} \mathrm{C}$ on standby. The procedures were performed according to the protocol. The samples were tested in the same batch and the intra-assay variation was $<5 \%$. Normal RBP reference range was $25-70 \mathrm{mg} / \mathrm{l}$, RBP level greater than normal value is positive. The newborns underwent cystoscopy for retrograde intubation. The renal urine was collected from the affected side. After centrifugation at $200 \mathrm{x} \mathrm{g}$ at room temperature for $5 \mathrm{~min}$, the supernatant was taken to adjust the $\mathrm{pH}$ 6.0-7.5. Level of Alb in the urine of the affected side was detected, the remaining urine samples were placed in a refrigerator at $-80^{\circ} \mathrm{C}$ on standby. Expression level of urinary Alb in the affected kidney was detected using ARRAY360 immunoprotein analyzer through immunoturbative nephelometry method. The kits were provided by Beckman Coulter, Inc., Brea, CA, USA. The operations were carried out in strict accordance with the instructions. All the samples were tested in the same batch, and the intra-assay variation was $<5 \%$. The normal value reference range of Alb was 0-40 mg/l and RBP level exceeding the normal value was considered positive.

Construction of a rat model of hydronephrosis. To investigate the diagnostic levels of Alb and RBP, we constructed a rat model of hydronephrosis. Thirty male SD rats (250-300 g) were purchased from our laboratory animal center [SYXK (Hei) 2015-007]. After adaptive feeding at room temperature for 1 week with the assurance of enough water and fodder, 30 rats were randomly divided into two groups: sham $(n=15)$ 
and unilateral ureteral obstruction (UUO) model $(n=15)$ groups. Surgery was performed after fasting for $12 \mathrm{~h}$. General anesthesia was performed by injecting $4 \%$ chloral hydrate into the abdominal cavity. After hair removal and disinfection, an incision was made in the middle of the abdomen. Ureter was ligated with a 1-0 silk thread $1 \mathrm{~cm}$ from the left ureteral junction and ligated again at a downstream distance of $1 \mathrm{~cm}$. After that, the incision was closed and intramuscular injection of penicillin sodium was performed to prevent wound infection. An incision was made in the middle of the abdomen after anesthesia, ureter was separated but not ligated in the Sham group. Model establishment was verified by color Doppler ultrasound 3 weeks later and the animals were sacrificed as indicated in a previous study (12). Kidneys of rats with hydronephrosis were taken for follow-up study, and the kidneys of Sham group were taken at the same position for comparative study.

Western blot analysis. Rat kidney was treated with protein lysate after grinding, and the protein in tissue was extracted using RIPA buffer (Sigma-Aldrich). Protein concentration was measured by BCA kit (Beyotime Institute of Biotechnology). After denaturation at $100^{\circ} \mathrm{C}$, marker $(5 \mu \mathrm{l}$ deep marker, $2 \mu \mathrm{l}$ shallow marker) and $40 \mathrm{mg}$ sample were added using a pipette. Electrophoresis was performed with $10 \%$ SDS page gel. After $60 \mathrm{~min}$ of electrophoresis, the protein was transferred to the PVDF membrane (Merck $\mathrm{KGaA}$ ) and sealed with $5 \%$ skimmed milk (BD Company) at room temperature for $60 \mathrm{~min}$. After gel transfer and blocking, membranes were washed, followed by incubation with the primary antibodies (RBP, Abcam, cat. no. ab190700, mouse monoclonal antibody, 1:1,000; Alb, Abcam, cat. no. ab207327, rabbit monoclonal antibody, 1:2,000; $\beta$-actin, Abcam, cat. no. ab8226, mouse monoclonal antibody, 1:500) overnight at $4^{\circ} \mathrm{C}$. After incubation with the corresponding secondary antibodies (Abcam, cat. no. ab6728, 1:2,000; Abcam, cat. no. ab6721, 1:1,000) for $2 \mathrm{~h}$, the membrane was scanned. Imaging was performed using special chemiluminescence colorant for ECL (Thermo Fisher Scientific, Inc.) and gel imaging image analysis system (Odyssey). ImageJ software (NIH) was used to analyze.

IL-12 treatment. Another 30 female SD rats (250-300 g) were randomly divided into the Sham group and IL-12 knockdown group, with 15 rats in each group. Sham group was given intravenous saline injection, while IL-12 knockdown group was given tail vein injection of IL-12 adenovirus, once every other day for one week. After the production of pregnant rats, the expression of RBP and Alb in kidneys of the two groups of offspring rats was detected.

Statistical analysis. A database was established in Excel. The measurement data were expressed as mean \pm standard deviation (mean $\pm \mathrm{SD}$ ). Data comparisons were performed by t-test. Data were statistically processed by Graphpad software, $\mathrm{P}<0.05$ indicated a difference with statistical significance. The correlation analysis was performed using the Pearson's correlation analysis. When $\mathrm{P}<0.05, \mathrm{r}$ absolute value $<0.5$ indicated low correlation, $>0.5, \mathrm{r}-<0.8$ absolute value was moderate correlation, and absolute value of $r>0.8$ was close correlation.
Table I. Comparison of RBP and Alb levels between the two groups of newborns.

\begin{tabular}{|c|c|c|c|c|}
\hline Items & Control $(n=24)$ & $\begin{array}{l}\text { Hydronephrosis } \\
\qquad(n=24)\end{array}$ & t value & P-value \\
\hline $\operatorname{RBP}(\mathrm{mg} / \mathrm{l})$ & $57.02 \pm 18.74$ & $95.36 \pm 30.87$ & 5.050 & 0.009 \\
\hline Alb (mg/l) & $29.24 \pm 7.47$ & $43.99 \pm 8.73$ & 6.153 & 0.013 \\
\hline
\end{tabular}

Table II. Comparison of positive rate of RBP and Alb detection in neonates with hydronephrosis.

\begin{tabular}{lccc}
\hline Items & Negative & Positive & Positive rate \\
\hline Combined detection & 1 & 23 & $95.83 \%$ \\
RBP $(\mathrm{mg} / \mathrm{l})$ & 4 & 20 & $83.33 \% \%^{\mathrm{a}}$ \\
Alb $(\mathrm{mg} / \mathrm{l})$ & 5 & 19 & $79.17 \%^{\mathrm{a}}$ \\
\hline
\end{tabular}

${ }^{\text {ap }}<0.05$ compared with combined detection.

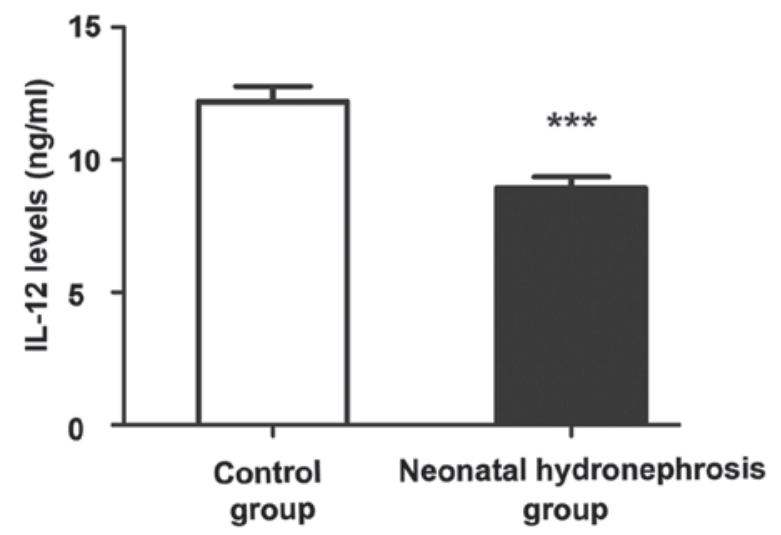

Figure 1. IL-12 levels in mothers of neonates with hydronephrosis and controls. ${ }^{* * *} \mathrm{P}=0.001$, compared with the control group.

\section{Results}

Detection of neonatal RBP and Alb. Samples of neonatal hydronephrosis patients and normal controls were collected to detect the expression of RBP and Alb. Results showed that expression levels of RBP and Alb in neonatal hydronephrosis patients were higher than those in healthy control group $(\mathrm{P}<0.05)$ (Table I).

Comparison of positive rate of $R B P$ and Alb detection in neonates with hydronephrosis. In 24 neonates with hydronephrosis, the positive rate of the single test was significantly lower than that of the RBP and Alb combined detection, and the difference was statistically significant $(\mathrm{P}<0.05)$ (Table II).

Comparisons of IL-12 levels in mothers of neonates with hydronephrosis and controls. Levels of IL-12 in mothers of 24 neonates with hydronephrosis and 24 healthy controls were compared. Compared with the control group, level of IL-12 in neonatal hydronephrosis group was significantly lower $(\mathrm{P}=0.001)$ (Fig. 1). 
Table III. Correlation analysis of nephridial hydronephrosis and levels of RBP, Alb and IL-12.

\begin{tabular}{lrrr}
\hline Neonatal hydronephrosis & RBP & Alb & IL-12 \\
\hline r value & 0.82 & 0.65 & -0.85 \\
P-value & $<0.05$ & $<0.05$ & $<0.05$ \\
\hline
\end{tabular}

\section{CTL UUO}
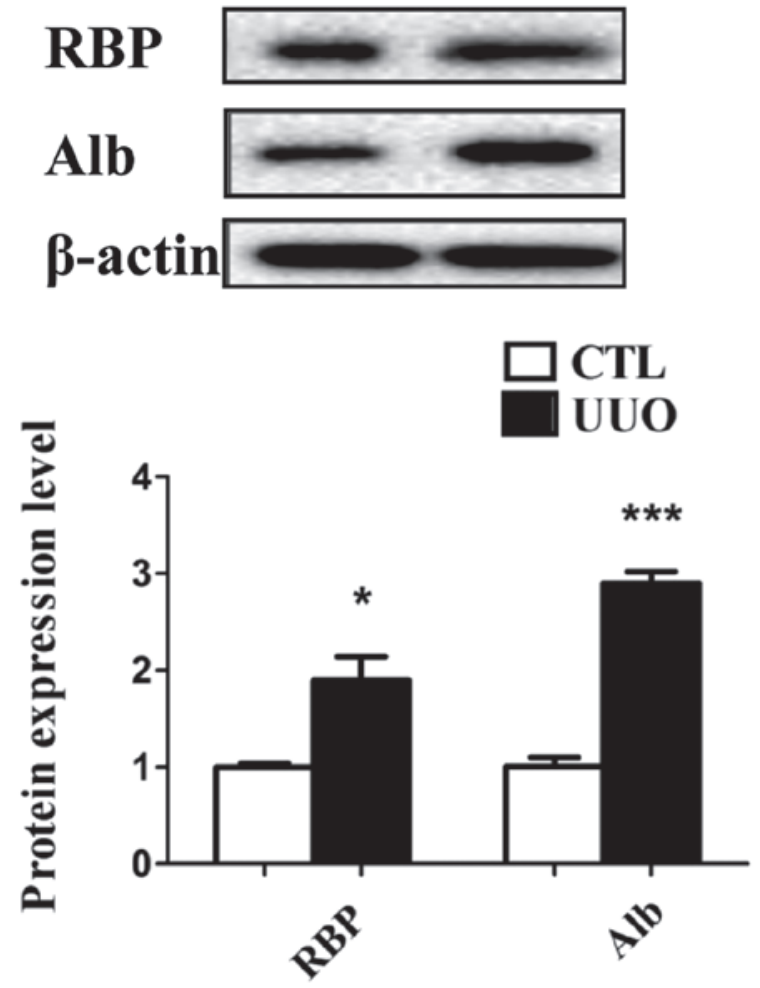

Figure 2. Expression levels of RBP and Alb in a rat model of hydronephrosis ${ }^{*} \mathrm{P}<0.05,{ }^{* * *} \mathrm{P}=0.001$, compared with the control group.

Relationship between the occurrence of neonatal hydronephrosis and levels of RBP and Alb and IL-12 levels in pregnant women before birth. Occurrence of neonatal hydronephrosis was highly positively correlated with RBP $(r=0.82, \mathrm{P}<0.05)$ and moderately positively correlated with Alb levels $(r=0.65$, $\mathrm{P}<0.05)$. There was a high negative correlation between the occurrence of hydronephrosis and IL-12 levels in their mothers before birth $(\mathrm{r}=-0.85, \mathrm{P}<0.05)$ (Table III).

Expression of RBP and Alb in a rat model of hydronephrosis. We examined the expression of RBP and Alb in the Sham and unilateral ureteral obstruction (UUO) model groups. The results of western blot analysis showed that, compared with control group, expression levels of RBP and Alb in model of hydronephrosis were increased, and the difference was statistically significant $(\mathrm{P}<0.05$ or $\mathrm{P}=0.001 ;$ Fig. 2$)$.

Expression of $R B P$ and Alb in offspring rats delivered by pregnant rats with IL-12 knockdown. Expression of RBP and $\mathrm{Alb}$ in offspring rats delivered by pregnant rats in Sham group
Table IV. Comparison incidence of hydronephrosis in offspring rats of Sham group and the IL-12 knockdown group.

\begin{tabular}{lccc}
\hline Groups $(\mathrm{n}=15)$ & Cases & $\begin{array}{c}\text { Cases of } \\
\text { hydronephrosis }\end{array}$ & $\begin{array}{c}\text { Incidence rate of } \\
\text { hydronephrosis }\end{array}$ \\
\hline Sham & 126 & 3 & $2.38 \%$ \\
IL-12 knockdown & 130 & 63 & $48.46 \%$ \\
\hline
\end{tabular}

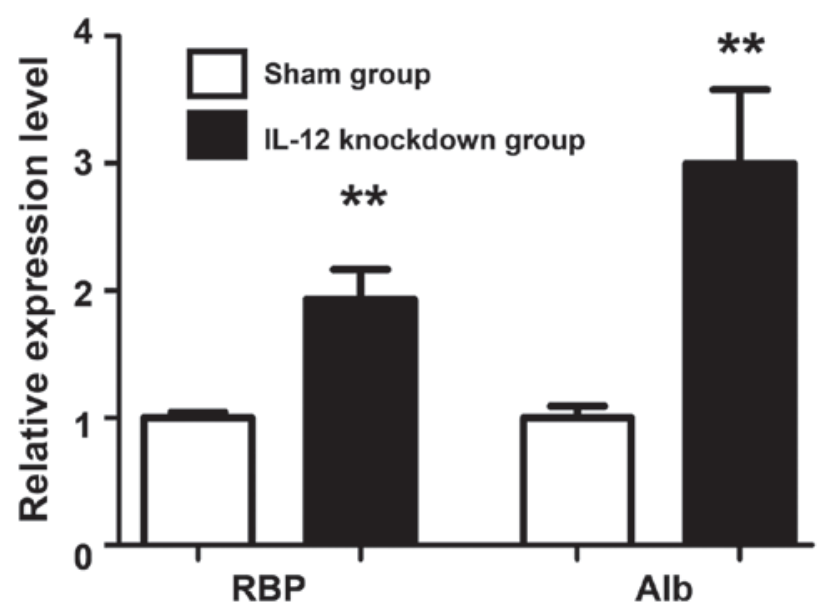

Figure 3. Expression of RBP and Alb in offspring rats delivered by pregnant rats with IL-12 knockdown. ${ }^{* *} \mathrm{P}<0.05$, compared to the control group.

and IL-12 knockdown group was detected. Results of western blot analysis showed that the expression of RBP and Alb in the kidney of the IL-12 knockdown group was significantly higher than that in the Sham group, and the difference was statistically significant $(\mathrm{P}=0.0056, \mathrm{P}=0.0099$, Fig. 3$)$. Results showed that the expression of RBP and Alb in offspring rats delivered by pregnant rats with IL-12 knockdown was significantly increased. The two groups of offspring rats were raised to reach body weight of 250-300 g, the color Doppler ultrasound of small animals was used to detect hydronephrosis. Results showed that compared with control group, IL-12 knockdown led to significantly increased incidence of hydronephrosis in offspring rats $(\mathrm{P}=0.001$, Table IV).

\section{Discussion}

Hydronephrosis is one of the common diseases in pediatric surgery. Obstruction of the urinary tract in any part of the urinary system can cause hydronephrosis. Congenital malformations, stones, tumors and inflammation can cause obstruction of the urinary tract (13). Hydronephrosis occurs mostly in children and newborns and the incidence is higher in men than in women. Unilateral hydronephrosis accounts for $80 \%$ of cases of hydronephrosis. Neonatal hydronephrosis seriously affects the health of newborns, and it also imposes a heavy burden on the family. Early detection and treatment can effectively control the progression of hydronephrosis, so early diagnosis and diagnosis of hydronephrosis is particularly important. At present, $99 \mathrm{mTc}$-diethylenetriamine pentaacetic acid (99mTc-DTPA) renal dynamic imaging is the most 
commonly used method for diagnosing neonatal hydronephrosis. This method has high requirements on the instrument and has certain radioactivity (14). Therefore, finding safer, faster and more accurate diagnostic indicators has become an important issue to be resolved in clinical and basic research.

In this study, we found that RBP and Alb can be used as diagnostic indicators for neonatal hydronephrosis, and the combined detection of RBP and Alb has important clinical significance in the diagnosis of hydronephrosis in neonates. Results of ELISA showed that the serum RBP and the renal urinary Alb level in neonates with hydronephrosis were higher than those in control group, the difference was statistically significant $(\mathrm{P}<0.05)$. RBP was highly correlated with neonatal hydronephrosis and Alb is moderately correlated with neonatal hydronephrosis. Compared with the combined detection of RBP and Alb, the positive rate of the single test was significantly lower, and the difference was statistically significant $(\mathrm{P}<0.05)$. In addition, we explored the relationship between the occurrence of neonatal hydronephrosis and IL-12 levels in pregnant women before birth. Results showed that level of IL-12 in the neonatal hydronephrosis group was lower than that in control group, and the difference was statistically significant $(\mathrm{P}<0.05)$. Level of IL-12 in pregnant women before birth is highly negatively correlated with neonatal hydronephrosis. In addition, we found that the expression of RBP and $\mathrm{Alb}$ protein was significantly elevated in the kidney of the rat model of hydronephrosis. Then IL-12 adenovirus treatment was performed on pregnant rats. The expression of RBP and Alb protein was detected after giving birth. We found that IL-12 knockdown caused a significant increase in the expression of $\mathrm{RBP}$ and Alb proteins in the kidneys of the offspring rats.

Liu et al (15) found that serum markers such as retinolbinding protein (RBP), $\beta 2$ microglobulin ( $\beta 2-\mathrm{MG})$, serum creatinine (Scr) and urea nitrogen (BUN) can be used to diagnose the sensitivity of different degrees of obstructive hydronephrosis and predict the accuracy of prognosis of renal function. Under normal circumstances, content of Alb in human urine is extremely low. When glomerular filtration membrane is abnormal, filtration excess of Alb is greater than the reabsorption of Alb. In this case, content of Alb in the urine is remarkably increased. RBP is a low molecular weight lipophilic carrier protein. In general, the expression level of RBP in urine in normal people is low. In the case of renal function damage, the content of RBP in urine is significantly increased. In normal human plasma, approximately $90 \%$ of RBP can specifically bind to thyroid binding protein, which is difficult to be filtered out by glomerulus, while the remaining $10 \%$ of RBP can be reabsorbed by renal tubules in the free-state after glomerular filtration. However, when the renal tubular reabsorption function is impaired, RBP in the human urine is significantly increased, and renal function of the human body can be directly evaluated. Therefore, we believe that children with neonatal hydronephrosis may have renal tubular reabsorption dysfunction, glomerular filtration membrane abnormalities, renal collecting duct obstruction, which may be related to different degrees of infection and inflammation in the later stages of pregnancy. Therefore, we believe that clinically, we can predict the possibility of neonatal hydronephrosis by detecting IL-12 levels during pregnancy to provide the important evidence for the diagnosis and treatment of hydronephrosis in neonates.
In summary, compared with previous studies, this study combined the detection of RBP and Alb levels in neonates with hydronephrosis, revealing that RBP and Alb combined detection has important clinical significance in the diagnosis of hydronephrosis in neonates. This study was the first to find that the occurrence of neonatal hydronephrosis may be related to the level of IL-12 in pregnant women before birth. Further studies will be needed with more participants to explore the relationship between IL-12 in pregnant women and the occurrence of hydronephrosis in neonates and to investigate whether the combined detection of RBP and Alb can serve as the critical value for markers of hydronephrosis in neonates of each stage so as to provide scientific basis for early diagnosis, prediction and treatment of neonatal hydronephrosis.

\section{Acknowledgements}

Not applicable.

\section{Funding}

This study was supported by Mudanjiang Science and Technology Plan (no. 201609202022).

\section{Availability of data and materials}

The datasets used and/or analyzed during the present study are available from the corresponding author on reasonable request.

\section{Authors' contributions}

ZM and DL performed ELISA and western blot analysis. WZ and $\mathrm{HW}$ were responsible for construction of rat model. ZM wrote the manuscript. All authors read and approved the final manuscript.

\section{Ethics approval and consent to participate}

The study was approved by the Ethics Committee of Hongqi Hospital Affiliated to Mudanjiang Medical University (Mudanjiang, China). Signed informed consents were obtained from the patients or the guardians.

\section{Patient consent for publication}

Not applicable.

\section{Competing interests}

The authors declare that they have no competing interests.

\section{References}

1. Goldberg H, Bachar GN, Majadla R, Yossepowitch O, Baniel J and Ram E: How prevalent is hydronephrosis secondary to acute appendicitis: A cross-sectional study. Isr Med Assoc J 20: 141-144, 2018.

2. Jiang D, Chen Z, Lin H, Xu M and Geng H: Predictive factors of contralateral operation after initial pyeloplasty in children with antenatally detected bilateral hydronephrosis due to ureteropelvic junction obstruction. Urol Int 100: 322-326, 2018 
3. Matsubara S, Morita T, Muto S and Suzuki T: Intrauterine balloon tamponade and bilateral hydronephrosis: Do they really have a cause-effect relationship? J Obstet Gynaecol 38: 587, 2018.

4. Li W, Zhao S, Cheng F, Rao T, Yu W, Ruan Y, Yuan R and Yao X: Oxidative damage and mitochondrial injuries differ following pneumoperitoneum pressure in rabbit models of varying degrees of hydronephrosis. Mol Med Rep 17: 6819-6827, 2018.

5. Baas W, O'Connor B and El-Zawahry A: Bilateral hydronephrosis and acute kidney injury secondary to pelvis lipomatosis. Can J Urol 25: 9217-9219, 2018.

6. Melvin JE, Ost MC and Marin JR: Hydronephrosis from ureteropelvic junction obstruction discovered on point-of-care ultrasound in patients with trauma. Pediatr Emerg Care 34: 365-367, 2018

7. Sobotka R, Čapoun O, Kalousová M, Hanuš T, Zima T, Koštíŕová M and Soukup V: Prognostic importance of vitamins A, $\mathrm{E}$ and retinol-binding protein 4 in renal cell carcinoma patients. Anticancer Res 37: 3801-3806, 2017.

8. Liu DY, Chen QZ and Liu GM: The changes in retinol-binding protein, N-acetyl-beta-glucosaminidase, albumin and IgG in urine before and after ESWL. Zhonghua Wai Ke Za Zhi 32: 570-572, 1994 (In Chinese).

9. Fan J, Yan P, Wang Y, Shen B, Ding F and Liu Y: Prevalence and clinical significance of low T3 syndrome in non-dialysis patients with chronic kidney disease. Med Sci Monit 22: 1171-1179, 2016

10. Liu Y, Perez J, Hammer LA, Gallagher HC, De Jesus M, Egilmez NK and Russell MW: Intravaginal administration of interleukin 12 during genital gonococcal infection in mice induces immunity to heterologous strains of neisseria gonorrhoeae. mSphere 3: 3, 2018.
11. Grignon A, Filion R, Filiatrault D, Robitaille P, Homsy Y, Boutin $\mathrm{H}$ and Leblond $\mathrm{R}$ : Urinary tract dilatation in utero: Classification and clinical applications. Radiology 160: 645-647, 1986.

12. Yang L, Li Y, Gong R, Gao M, Feng C, Liu T, Sun Y, Jin M, Wang D, Yuan Y, Yan G, He M, Idiiatullina E, Ma W, Han Z, Zhang L, Huang Q, Ding F, Cai B and Yang F: The long noncoding RNA-ORLNC1 regulates bone mass by directing mesenchymal stem cell fate. Mol Ther 27: 394-410, 2019.

13. Wong MCY, Piaggio G, Damasio MB, Molinelli C, Ferretti SM, Pistorio A, Ghiggeri G, Degl'Innocenti ML, Canepa A, Incarbone V, et al: Hydronephrosis and crossing vessels in children: Optimization of diagnostic-therapeutic pathway and analysis of color Doppler ultrasound and magnetic resonance urography diagnostic accuracy. J Pediatr Urol 14: 68.e1-68.e6, 2018.

14. Ebel KD: Uroradiology in the fetus and newborn: Diagnosis and follow-up of congenital obstruction of the urinary tract. Pediatr Radiol 28: 630-635, 1998

15. Liu JN, Liu YD, Yang K and An R: Significance of biomarkers in the diagnosis and prognosis of hydronephrosis. J Clin Urol 33: 393-397, 2018 (In Chinese).

This work is licensed under a Creative Commons Attribution-NonCommercial-NoDerivatives 4.0 International (CC BY-NC-ND 4.0) License. 